\title{
Patient satisfaction with oral health check-ups at a community pharmacy and their effect on oral self-care habits and dental consultation behavior
}

\author{
Hiroki Iwata $^{1,2}$, Koichi Shibano ${ }^{1}$, Mitsuhiro Okazaki ${ }^{3,4}$, Kotaro Fujimaki ${ }^{5,6}$, Noriko Kobayashi, ${ }^{1,2}$, \\ Kazuko Fujimoto ${ }^{1}$, Naoko Hayashi, ${ }^{1,2}$, Tomoyuki Goto ${ }^{6,7}$, Katsunori Yamaura ${ }^{1,2, *}$ \\ ${ }^{1}$ Division of Social Pharmacy, Center for Social Pharmacy and Pharmaceutical Care Sciences, Faculty of Pharmacy, Keio University, Tokyo, Japan; \\ ${ }^{2}$ Keio University Community Pharmacy, Tokyo, Japan; \\ ${ }^{3}$ Department of Drug Policy \& Management, Graduate School of Pharmaceutical Sciences, The University of Tokyo, Tokyo, Japan; \\ ${ }^{4}$ Health Innovation \& Technology Center, Faculty of Health Sciences, Hokkaido University, Hokkaido, Japan; \\ ${ }^{5}$ Bubai Ohana Dental Clinic, Tokyo, Japan; \\ ${ }^{6}$ Shinjuku Shoku-Shien Kenkyukai, Tokyo, Japan; \\ ${ }^{7}$ Goto Dental Office, Tokyo, Japan.
}

SUMMARY Maintaining good oral health is important because oral diseases are related to systemic diseases, and community pharmacies play a key role in maintaining the health of local residents. This study aimed to examine the effects of oral health check-ups and information provision at community pharmacies on oral health-associated behaviors as well as patient satisfaction. We conducted oral health checkups and provided information about oral health self-care to 84 patients at a community pharmacy, and then asked them to complete a questionnaire survey. One month later, we sent them a followup questionnaire and received responses from $66.7 \%$ (56/84) of the participants. The large majority were satisfied with the salivary test $(95.2 \%)$ and the information $(96.4 \%)$ we provided. Most of the participants $(89.3 \%)$ indicated that they wanted to use the oral health check-up service again in the future. Compared with baseline, the ratio of participants restricting their intake of sugar-rich foods and drinks significantly increased 1 month later $(p=0.021)$. About $60 \%$ of those who had not undergone a regular dental examination at baseline reported newly visiting or planning to visit a dental clinic. The results revealed high satisfaction with the oral health check-up and information about oral selfcare they received at the community pharmacy. The results suggested that oral health check-ups had the potential to change both oral self-care habits and dental consultation behavior. Our findings indicate that community pharmacies can contribute to the maintenance and promotion of oral health by providing oral health check-ups to local residents.

Keywords health support, behavioral change, salivary test, information provision, dental clinic, questionnaire survey

\section{Introduction}

Oral diseases, such as dental caries and periodontal disease, are major public health problems worldwide $(1,2)$. In Japan, about 4 million patients were estimated to have periodontal disease, making it the second most common major disease behind hypertension (3). Oral diseases lead not only to loss of oral function but also to lower quality of life (4). In addition, it is known that periodontal disease is related to systemic diseases, including diabetes mellitus $(5,6)$ and atherosclerotic vascular disease (7). It has been reported that periodontal treatment is effective for improving glycemic control
(8) and that regular dental care utilization reduces the risk of stroke (9). Therefore, maintenance of oral health is important for prolonging health expectancy and decreasing national medical expenditures.

Regular dental check-ups are essential for early detection of oral diseases. However, only $52.9 \%$ of Japanese adults have regular dental check-ups according to a survey conducted by the Ministry of Health, Labour and Welfare in 2016 (10), and this is much lower than the rate of regular health check-ups (71.4\%) (11). Therefore, a strategy is needed to close this gap in behaviors.

Community pharmacies play an important role in maintaining the health of the local residents and 
prolonging their health expectancy. A majority of pharmacists in Australia $(12,13)$ and England (14) stated that it was their role to deliver oral health advice to the community and provide oral healthcare service to local residents. However, Japanese community pharmacists merely advise patients about the adverse effects of medicine in the oral cavity (15), and few studies have investigated the contribution of community pharmacists to the oral healthcare of local residents. In September 2015, the Ministry of Economy, Trade and Industry allowed salivary tests to be used to conduct oral health check-ups at community pharmacies (16). This oral health check-up could become a new tool for community pharmacies to maintain and promote the oral health of local residents. However, very few Japanese community pharmacies have introduced this service. Therefore, in the present study, we conducted oral health checkups and provided information about oral self-care at a community pharmacy and examined the effect on oral health-associated behaviors and conducted surveys to determine satisfaction as well as how likely participants were to use the service again in the future.

\section{Materials and Methods}

\subsection{Participants and study design}

Participants were recruited from among patients who visited Keio University Community Pharmacy (Tokyo, Japan) with a prescription in June or July 2018. The researchers explained the details of the study to each patient and obtained written informed consent prior to their participation. Individuals under 20 years old were excluded. The oral health condition of the participants was checked by using a salivary test, which was free of charge, and each participant was given their results sheet. In addition, the participants received information about oral health self-care from the researchers and were asked to respond to a self-administered questionnaire. One month later, the participants were asked to respond to a follow-up questionnaire sent to their home by postal mail. The study protocol was approved by the research ethics committee of the Faculty of Pharmacy, Keio University (approval number: 180528-1).

\subsection{Oral health check-up by salivary test}

The oral health check-up was conducted using a salivary test system (SillHa; ARKRAY Inc., Kyoto, Japan), which assays the following six items in about 5 min: cariogenic bacteria, $\mathrm{pH}$, buffer capacity (which indicates the risk of dental caries), leukocytes, protein (which indicates the risk of periodontal disease), and ammonia (which indicates oral cleanliness). This test cannot be used to diagnose oral diseases. In this study, participants provided oral rinse sample using $3 \mathrm{~mL}$ of distilled water, and the researchers used a dropper to transfer the sample onto the test strip. Once the assay was complete, the researchers explained the results to the participant, referencing a results sheet, on which the results of the six items were plotted on a radar chart. The participants were also told that the results were often affected by their diet and oral hygiene.

\subsection{Information provision about oral health}

The researchers gave each participant an original leaflet containing information about the relationship between oral diseases and systemic diseases, recommendations for dietary behaviors and oral self-care, and an explanation on the importance of regular dental checkups, and they discussed the contents of the leaflet with each participant for a few minutes. Regarding dietary behaviors, the researchers recommended that the participants restrict their intake of sugar-rich foods and drinks, eat nutritionally balanced meals, and chew their meals wells. In addition, the researchers provided oral hygiene guidance, including the use of an interdental brush or dental floss as well as fluoride-containing toothpaste. Among the researchers were two dentists, who contributed to the creation of the information leaflet.

\subsection{Questionnaire survey}

At the end of the session at the community pharmacy, each participant was asked to complete a selfadministered questionnaire covering the following areas: 1) basic characteristics and frequency of regular dental check-ups, 2) dietary behaviors and oral hygiene habits in daily life, and 3) satisfaction with the oral health check-up and information provided and their likelihood of using the service again in the future.

One month later, participants were asked to respond to a follow-up questionnaire sent to their home by postal mail, which asked about dietary behaviors, oral hygiene habits, and dental consultation behavior.

\subsection{Statistical analysis}

All analyses were performed using IBM SPSS Statistics version 25 software (IBM Corp., Armonk, NY), and McNemar's test was used to evaluate changes between baseline and 1 month later. A $p$-value $<0.05$ was regarded as significant.

\section{Results}

\subsection{Participant characteristics}

A total of 84 patients participated in this study; their basic characteristics are shown in Table 1 . Most participants (32.1\%) were in their 70s and just over half $(53.6 \%)$ were women. Only $9.5 \%$ were smokers. Nearly 
two-thirds of the participants $(64.3 \%)$ had undergone a regular dental check-up in the preceding 12 months and the rest $(35.7 \%)$ had not.

3.2. Satisfaction with the oral health check-up and the information provided at the community pharmacy

Most participants (77.4\%) found it beneficial to get

Table 1. Participant characteristics $(n=84)$

\begin{tabular}{lc}
\hline & $n(\%)$ \\
\hline Age & \\
$20-29$ & $4(4.8)$ \\
$30-39$ & $4(4.8)$ \\
$40-49$ & $12(14.3)$ \\
$50-59$ & $18(21.4)$ \\
$60-69$ & $14(16.7)$ \\
$70-79$ & $27(32.1)$ \\
$80+$ & $5(6.0)$ \\
Sex & \\
Male & $39(46.4)$ \\
Female & $45(53.6)$ \\
Smoking habit & \\
Yes & $8(9.5)$ \\
No & $76(90.5)$ \\
Regular dental check-up in the preceding 12 months & \\
Yes & $54(64.3)$ \\
No & $30(35.7)$ \\
\hline
\end{tabular}

their results on site after the oral health check-up at a community pharmacy (Figure 1A). Additionally, they said that the oral health check-up was easy $(66.7 \%)$, was useful for improving their oral self-care habits $(56.0 \%)$, and took a short time $(51.2 \%)$. Furthermore, $79.8 \%$ of participants answered that there was no demerit about having the oral health check-up at a community pharmacy. Only a few participants felt that the presence of dental caries and periodontal disease were not confirmed by the oral health check-up (4.8\%) and hesitated to provide a saliva sample for the assay (3.6\%).

Nearly all the participants $(95.2 \%)$ indicated that they were "satisfied" or "somewhat satisfied" with the oral health check-up at the community pharmacy (Figure 1B) and $96.4 \%$ indicated that they were "satisfied" or "somewhat satisfied" with the information they received about oral health self-care (Figure 1C). No one responded that they were "somewhat unsatisfied" or "unsatisfied" about either the oral health check-up or the information provided. Moreover, $89.3 \%$ of the participants indicated that they wanted to use the oral health check-up service at a community pharmacy again in the future (Figure 1D).

3.3. Changes in oral self-care habits and dental consultation behavior 1 month after the oral health check-up

(A)

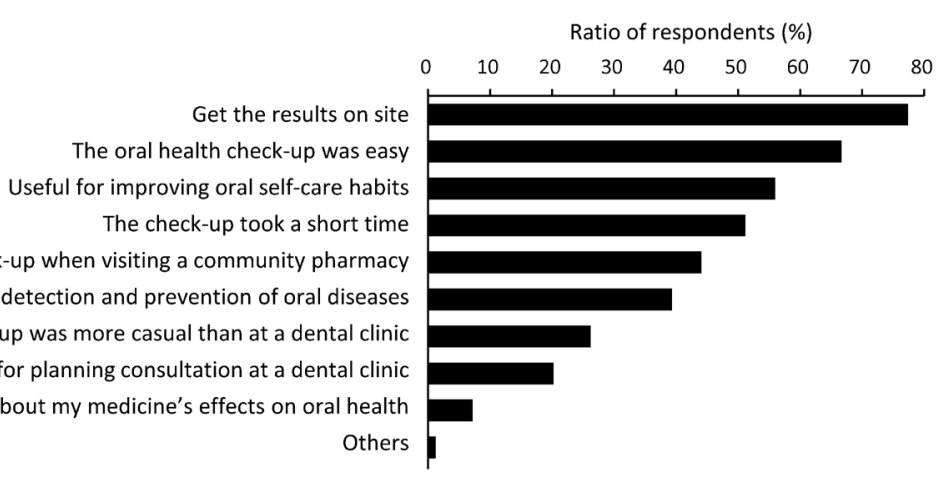

(B)

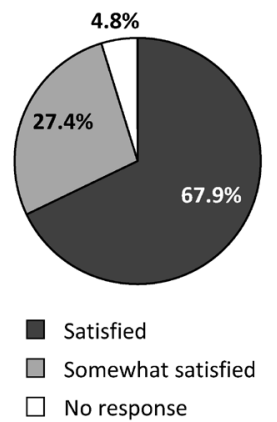

(C)

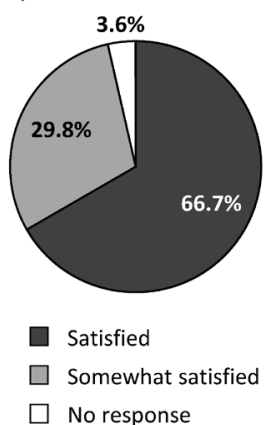

(D)

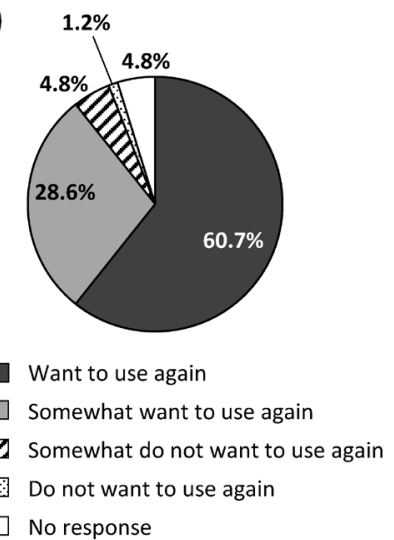

Figure 1. Satisfaction with the oral health check-up and the information provided at the community pharmacy. (A) Benefits of the oral health check-ups performed at the community pharmacy ( $n=84$, multiple answers allowed). (B and C) Degree of satisfaction with (B) the oral health check-up and (C) the information provided about oral self-care at the community pharmacy $(n=84)$. (D) Likelihood of using the oral health check-up service at a community pharmacy again $(n=84)$. 
(A)

Ratio of respondents (\%)

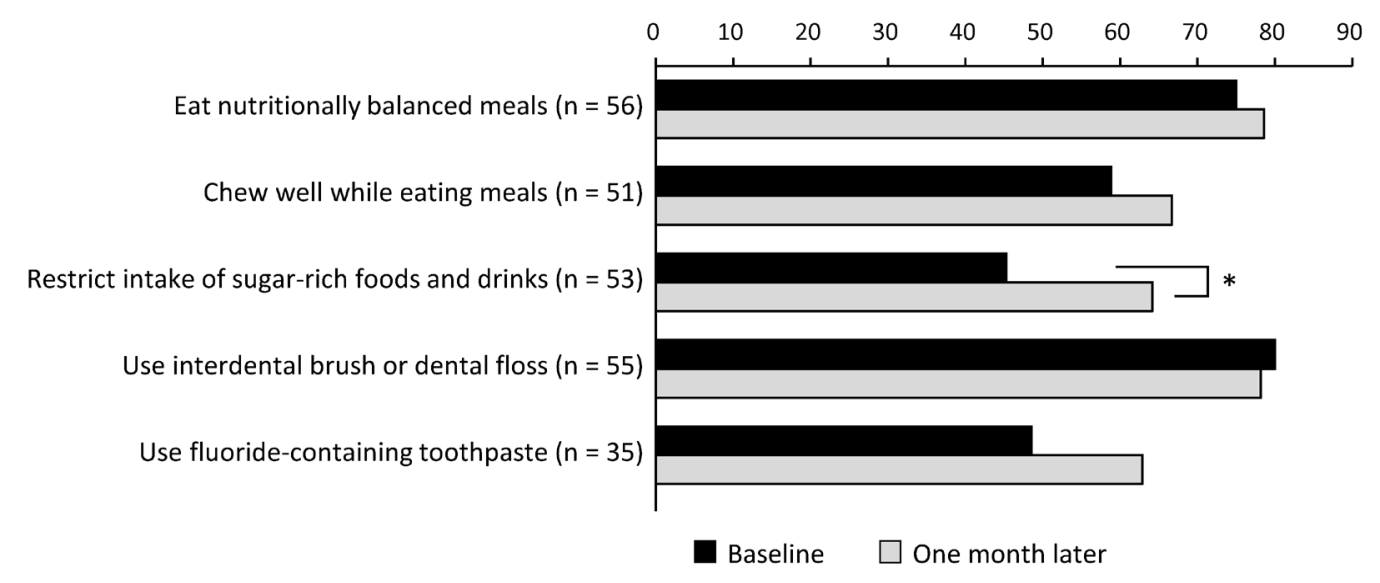

(B)

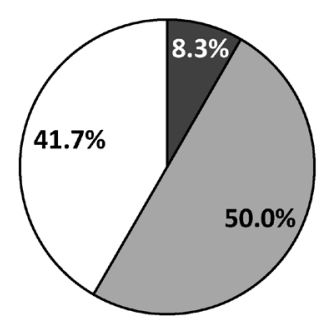

Newly visited a dental clinic

Planning to visit a dental clinic

Not planning to visit a dental clinic

Figure 2. Changes in oral self-care habits and dental consultation behavior 1 month after the oral health check-up. (A) Comparison of oral self-care habits at baseline and 1 month after the oral health check-up performed at the community pharmacy. Data from respondents were excluded if they did not answer the same items on both questionnaires and if they answered "I don't know if my toothpaste contains fluoride" to the item on use of fluoride-containing toothpaste. The number of responses for each item are indicated in the figure. ${ }^{*} p<0.05$ by McNemar's test. (B) Dental consultation behavior 1 month after the oral health check-up performed at the community pharmacy. Figure shows data of those who had not gone to a regular dental check-up in the preceding 12 months at baseline and responded to the follow-up questionnaire 1 month later $(n=12)$.

We received responses from $66.7 \%(56 / 84)$ of the participants to the follow-up questionnaire we administered 1 month after the oral health check-up at the community pharmacy. The ratio of participants who performed various aspects of oral self-care at the time of the oral health check-up (baseline) to those 1 month later is shown in Figure 2A. More than $70 \%$ of the participants ate nutritionally balanced meals and used an interdental brush or dental floss at baseline, whereas less than 50\% restricted their intake of sugar-rich foods and drinks and used a fluoride-containing toothpaste. Compared with baseline, the ratio of participants restricting their intake of sugar-rich foods and drink had significantly increased 1 month later (baseline, $45.3 \%$; 1 month later, $64.2 \% ; p$ $=0.021$ ).

Of the 56 participants who responded to the followup questionnaire, 12 had not gone to a regular dental check-up in the preceding 12 months. Only one of them $(8.3 \%)$ reported newly visiting a dental clinic during the month after they used the oral health check-up service at a community pharmacy, whereas half indicated plans to visit a dental clinic in the near future (Figure 2B).

\section{Discussion}

The results of this study showed that the majority of participants were satisfied with the oral health checkup service conducted using a salivary test as well as the information provided about oral self-care and indicated that they wanted to use the service again in the future. In addition, some of the participants reported making positive changes in their oral self-care habits and dental consultation behavior. These results suggest that providing oral health check-up services at community pharmacies could lead to improvements in oral healthassociated behavior in local residents.

This is the first study to investigate oral health checkup services at a Japanese community pharmacy in terms of effectiveness, satisfaction, and likelihood of using the service again in the future. Compared with other countries (12-14), Japanese community pharmacies are less proactive in promoting and maintaining the oral health of local residents. The introduction of this new service comprising an oral health check-up and provision of oral self-care information is expected to help Japanese community pharmacies take a more active role in promoting and maintaining the oral health of local residents.

The results of this study showed that both the oral health check-up and the information provided about oral self-care were very well received by the participants, many of whom recognized the ease and speed with which 
they could receive both the check-up and the results. In addition, the check-up procedure does not cause pain or distress because users need only provide an oral rinse sample. Furthermore, the assay takes only $5 \mathrm{~min}$ to complete, so it should generally be possible to complete the oral health check-up while the user is waiting for their prescription to be filled. Also, given that the large majority of participants indicated that they wanted to use the oral health check-up service again in the future, it is recommended that more Japanese community pharmacies introduce this service to accommodate the expected demand.

More than half the participants indicated that the oral health check-up and the information they received were useful for improving their oral self-care habits. Indeed, the ratio of participants restricting their intake of sugarrich foods and drinks significantly increased 1 month after the check-up compared with baseline, suggesting that the check-up and information provision might have led to changes in their oral health-associated behaviors.

The results also suggest that the service affected the participants' dental consultation behavior because about $60 \%$ of participants who had not gone to a regular dental check-up in the preceding 12 months reported newly visiting a dental clinic or planning to do so in the near future. In this study, the participants were recommended to visit a dental clinic for a professional examination and care during the oral health check-up session. Given that oral diseases are known to be related to systemic diseases (5-7), it is possible that the oral health check-up conducted at community pharmacies possibly led not only to early detection of oral diseases but also to the prevention of systemic diseases.

From the results of the follow-up questionnaire survey conducted 1 month after the oral health checkup, no significant improvements were observed in chewing well while eating meals or using fluoridecontaining toothpaste, and the positive effect on dental consultation behavior was limited. Regarding the effects of intervention at community pharmacies, previous studies found that repeated implementation of lifestyle advice and measurements led to reductions in blood pressure (17) and improvements in bone mineral density (18) in local residents. Although we conducted the oral health check-ups and provided information only one time, it is expected that subsequent sessions would lead to further changes in oral self-care habits and dental consultation behavior.

A limitation of this study is that we evaluated only a combination group in which both the oral health check-up was performed and the information was provided and there was no control group. Further study is required to more clearly assess the effects of the oral health check-up and information provision separately.

In conclusion, the present study demonstrated that participants were very satisfied with the oral health check-up and the information provided about oral self- care at the community pharmacy and that they wanted to use the service again in the future. Additionally, it was suggested that the oral health check-up and information provision potentially led to improvements in oral self-care and dental consultation behavior. These findings indicate that community pharmacies could possibly contribute to the promotion and maintenance of oral health in local residents by providing this service.

\section{Acknowledgements}

We are grateful to the individuals who participated in this study.

Funding: This work was supported by the Keio University Academic Development Funds for Individual Research, a grant from the OTC Self-Medication Promotion Foundation, and a JSPS KAKENHI Grantin-Aid for Scientific Research (C; Grant Number JP20K07185).

Conflict of Interest: The authors have no conflicts of interest to disclose.

\section{References}

1. Bernabe E, Marcenes W, Hernandez CR, et al. Global, regional, and national levels and trends in burden of oral conditions from 1990 to 2017: A systematic analysis for the global burden of disease 2017 study. J Dent Res. 2020; 99:362-373.

2. Petersen PE, Ogawa H. The global burden of periodontal disease: towards integration with chronic disease prevention and control. Periodontol 2000. 2012; 60:15-39.

3. Ministry of Health, Labour and Welfare in Japan. Patient Survey 2017. https://www.mhlw.go.jp/toukei/saikin/hw/ kanja/17/dl/kanja-01.pdf (accessed October 18, 2020). (in Japanese)

4. Buset SL, Walter C, Friedmann A, Weiger R, Borgnakke WS, Zitzmann NU. Are periodontal diseases really silent? A systematic review of their effect on quality of life. J Clin Periodontol. 2016; 43:333-344.

5. Ide R, Hoshuyama T, Wilson D, Takahashi K, Higashi T. Periodontal disease and incident diabetes: a seven-year study. J Dent Res. 2011; 90:41-46.

6. Taylor GW. Bidirectional interrelationships between diabetes and periodontal diseases: an epidemiologic perspective. Ann Periodontol. 2001; 6:99-112.

7. Aarabi G, Eberhard J, Reissmann DR, Heydecke G, Seedorf U. Interaction between periodontal disease and atherosclerotic vascular disease - Fact or fiction? Atherosclerosis. 2015; 241:555-560.

8. Sgolastra F, Severino M, Pietropaoli D, Gatto R, Monaco A. Effectiveness of periodontal treatment to improve metabolic control in patients with chronic periodontitis and type 2 diabetes: a meta-analysis of randomized clinical trials. J Periodontol. 2013; 84:958-973.

9. Sen S, Giamberardino LD, Moss K, Morelli T, Rosamond WD, Gottesman RF, Beck J, Offenbacher S. Periodontal disease, regular dental care use, and incident ischemic 
stroke. Stroke. 2018; 49:355-362.

10. Ministry of Health, Labour and Welfare in Japan. National Health and Nutrition Survey 2016. https://www. mhlw.go.jp/bunya/kenkou/eiyou/dl/h28-houkoku-06.pdf (accessed October 18, 2020). (in Japanese)

11. Ministry of Health, Labour and Welfare in Japan. National Health and Nutrition Survey 2018. https://www.mhlw. go.jp/content/000615345.pdf(accessed October 18, 2020). (in Japanese)

12. Freeman CR, Abdullah N, Ford PJ, Taing MW. A national survey exploring oral healthcare service provision across Australian community pharmacies. BMJ Open. 2017; 7:e017940.

13. Taing MW, Ford PJ, Gartner CE, Freeman CR. Describing the role of Australian community pharmacists in oral healthcare. Int J Pharm Pract. 2016; 24:237-246.

14. Mann RS, Marcenes W, Gillam DG. Is there a role for community pharmacists in promoting oral health? Br Dent J. 2015; 218:E10.

15. Ueda M, Niki K, Ueda H, Oishi M, Uejima E. The need for medical, dental and pharmacological cooperation focused on the oral cavity functions - The role of community pharmacy on appropriate pharmacotherapy for the prevention of serious adverse effects on the oral cavity. Jpn J Pharm Health Care Sci. 2017; 43:320-327. (in Japanese).

16. Ministry of Economy, Trade and Industry in Japan. News Release (September 1, 2015) - Consciousness of the implementation of oral health check-ups with salivary tests at community pharmacies. https://www.meti.go.jp/ policy/jigyou_saisei/kyousouryoku_kyouka/shinjigyokaitakuseidosuishin/press/150901_press.pdf (accessed October 18, 2020). (in Japanese)

17. Okada H, Onda M, Shoji M, Sakane N, Nakagawa Y, Sozu T, Kitajima Y, Tsuyuki RT, Nakayama T. Effects of lifestyle advice provided by pharmacists on blood pressure: The community pharmacists assist for blood pressure (COMPASS-BP) randomized trial. Biosci Trends. 2017; 11:632-639.

18. Wada Y, Wada Y, Ennyu S, Shimokawa KI, Ishii F. Ability of community pharmacists to promote self-care and selfmedication by local residents [I]: Improvements in bone mineral density. Drug Discov Ther. 2017; 11:35-40.

Received October 24, 2020; Revised December 3, 2020; Accepted December 12, 2020.

*Address correspondence to:

Katsunori Yamaura, Division of Social Pharmacy, Center for Social Pharmacy and Pharmaceutical Care Sciences, Faculty of Pharmacy, Keio University, 1-5-30 Shibakoen, Minato-ku, Tokyo 105-8512, Japan.

E-mail: yamaura-kt@keio.jp

Released online in J-STAGE as advance publication December 27, 2020. 